\title{
Patient Safety Climate in Healthcare Organizations: tradução e adaptação para a cultura brasileira
}

\author{
Patient Safety Climate in Healthcare Organizations: translation and adaptation to the \\ Brazilian culture \\ Patient Safety Climate in Healthcare Organizations: traducción y adaptación a la \\ cultura brasileña
}

\author{
Marcia Raquel Panunto Dias Cunha ${ }^{a}$ \\ Edinêis de Brito Guirardello ${ }^{b}$
}

\section{Como citar este artigo:}

Cunha MRPD, Guirardello EB. Patient

Safety Climate in Healthcare Organi-

zations: tradução e adaptação para a cultura brasileira. Rev Gaúcha Enferm. 2018:39:e20180010. doi: https://doi. org/10.1590/1983-1447.2018.20180010
Universidade Estadual de Campinas (UNICAMP), Colégio Técnico de Campinas, Departamento de Enfermagem. Campinas, São Paulo, Brasil.

' Universidade Estadual de Campinas (UNICAMP), Faculdade de Enfermagem. Campinas, São Paulo, Brasil.

\section{RESUMO}

Objetivo: Traduzir e adaptar o instrumento Patient Safety Climate in Healthcare Organizations - PSCHO para a cultura brasileira. Métodos: Estudo metodológico, o qual seguiu as etapas de tradução; síntese; retrotradução; avaliação por um comitê de juízes, composto por uma médica e seis enfermeiras; e pré-teste com 40 sujeitos, entre eles enfermeiros, técnicos de enfermagem, fisioterapeutas e médicos.

Resultados: As etapas de tradução e retrotradução foram apropriadas. Na síntese, consultou-se a autora do instrumento para ajustar a equivalência de alguns termos. A análise do comitê de juízes demonstrou um índice de validade de conteúdo acima de 80\% para a maioria dos itens quanto às equivalências semântica, idiomática, cultural e conceitual. No pré-teste, observou-se adequada validade de conteúdo, facilidade de compreensão dos itens, com um tempo médio de preenchimento de 15 minutos.

Conclusão: A tradução e adaptação cultural do instrumento PSCHO foram consideradas satisfatórias, resultando em uma ferramenta de fácil compreensão.

Palavras-chave: Enfermagem. Estudos de validação. Segurança do paciente.

\section{ABSTRACT}

Objective: To translate and adapt the Patient Safety Climate in Healthcare Organizations - PSCHO questionnaire to the Brazilian culture. Methods: Methodological study, which followed the stages of translation; synthesis; back-translation; evaluation by an experts committee, composed of one doctor and six nurses; and pretest with 40 subjects, among them nurses, nursing technicians, physiotherapists and physicians.

Results: The translation and back-translation steps were appropriate. In the synthesis step, the author of the questionnaire was consulted to adapt the equivalence of some terms. The analysis of the experts committee showed a content validity rate above $80 \%$ for most items regarding semantic, idiomatic, cultural and conceptual equivalence. In the pretest, it was observed appropriate content validity, items easy to understand, with an average application time of 15 minutes.

Conclusion: The translation and cultural adaptation of the PSCHO questionnaire was considered satisfactory, resulting in an easily understandable tool.

Keywords: Nursing. Validation studies. Patient safety.

\section{RESUMEN}

Objetivo: Traduciry adaptar el instrumento Patient Safety Climate in Healthcare Organizations - PSCHO a la cultura Brasileña.

Métodos: Estudio metodológico que siguió las etapas: traducción; síntesis; retrotraducción; evaluación por un comité de expertos, compuesto por una médica y seis enfermeras; y prueba previa con 40 sujetos, entre ellos enfermeros, técnicos de enfermería, fisioterapeutas y médicos.

Resultados: Las etapas de traducción y retrotraducción fueron apropiadas. En la síntesis, se consultó la autora del instrumento para ajustar la equivalencia de algunos términos. El análisis del comité de expertos demostró un índice de validez de contenido superior al $80 \%$ en la mayoría de los ítems, en cuanto a las equivalencias semánticas, idiomáticas, culturales y conceptuales. En el pre-test, se observó una adecuada validez de contenido, facilidad de comprensión de los ítems, con un tiempo medio de llenado de 15 minutos. Conclusión: La traducción y adaptación cultural del instrumento PSCHO se consideró satisfactoria, lo que resulta en una herramienta de fácil compresión.

Palabras clave: Enfermería. Estudios de validación. Seguridad del paciente. 


\section{—INTRODUÇÃO}

Os eventos relacionados à assistência à saúde ocupam a terceira causa de morte nos Estados Unidos e milhões de incidentes têm sido notificados ao redor do mundo, o que evidencia a necessidade de compreensão dos fatores que colocam o paciente em risco e à incorporação de medidas que contribuam para organizações de saúde mais seguras ${ }^{(1-2)}$. A busca por intervenções sistemáticas pode contribuir para a redução desses eventos e requer a construção de um processo de trabalho pautado num modelo de cultura de segurança ${ }^{(3)}$.

A cultura de segurança é definida como o produto de valores, atitudes, percepções, competências e padrões de comportamento individuais e coletivos ${ }^{(3)}$, a qual pode ser mensurada por meio do clima, uma medida temporal, onde se considera a percepção dos profissionais em relação ao comportamento e ações que priorizam a segurança em seu ambiente de trabalho ${ }^{(4)}$.

Há evidências da relação entre ambientes com cultura de segurança positiva e a redução das taxas de mortalidade, quedas, infecção e melhor satisfação do paciente ${ }^{(5-6)} \mathrm{e}$ o uso de ferramentas para avaliação do clima possibilita identificar as lacunas nas organizações de saúde para implementação de estratégias que fortaleçam a segurança. No Brasil, encontram-se disponíveis o Hospital Survey on Patient Safety Culture (HSOPSC) ${ }^{(7)}$ e o Safety Attitudes Questionnaire $(\mathrm{SAQ})^{(8)}$. Contudo, enquanto o HSOPSC apresenta alterações na sua estrutura dimensional apontadas em outros países, o que dificulta comparações ${ }^{(7)}$, estudos que utilizaram o SAQ carecem de dados de confiabilidade do instrumento.

Um outro instrumento com esta mesma finalidade, o Patient Safety Climate in Healthcare Organizations ${ }^{(9)}$, ainda não validado para a nossa cultura, mostra-se como uma ferramenta capaz de mensurar o clima de segurança das organização de saúde a partir da percepção dos profissionais, tanto daqueles que trabalham na assistência direta ao paciente, quanto dos que gerenciam a organização(10). A sua construção tem por base a teoria relacionada às características de Organizações de Alta Confiabilidade (High Reliability Organizations - HRO), que operam em condições perigosas e possuem uma porcentagem mínima de eventos adversos ${ }^{(11)}$. Entre os principais atributos dessas organizações estão: processos de trabalho bem articulados e resilientes; preocupação com falhas; resistência em aceitar a simplificação de interpretações, focando na compreensão das verdadeiras causas dos erros; sensibilidade para reconhecer precocemente indicadores que ameaçam o desempenho organizacional; e tomada de decisão por in- divíduos com maior conhecimento sobre os processos de trabalho, independente do cargo ou posição que ocupem dentro da organização(11).

Apesar da disponibilidade de instrumentos em nossa cultura para avaliação do clima de segurança, o PSCHO é uma ferramenta que, além dessa finalidade, possibilita identificar o percentual de respostas problemáticas em questões que influenciam a percepção dos profissionais, tanto na unidade de trabalho quanto na instituição, o que permite ao gerente implementar ações de melhorias de acordo com sua demanda. Dessa forma, o presente estudo teve como objetivo traduzir e adaptar o instrumento Patient Safety Climate in Healthcare Organizations para a cultura brasileira.

\section{—MÉTODO}

Trata-se de um estudo metodológico, caracterizado por processos de desenvolvimento e avaliação de instrumentos de coleta de dados, originado da tese intitulada "Adaptação cultural e avaliação das propriedades de medida do instrumento Patient Safety Climate in Healthcare Organizations"(12). A etapa apresentada a seguir foi conduzida entre junho de 2015 e maio de 2017, em uma instituição hospitalar pública de ensino do interior do Estado de São Paulo.

\section{Patient Safety Climate in Healthcare Organizations - PSCHO}

O PSCHO possui 51 itens, seis referentes às características profissionais e 45 sobre o clima de segurança, dos quais 42 estão distribuídos em 12 dimensões que mensuram aspectos do hospital, da unidade de trabalho e interpessoais sobre o clima de segurança. As dimensões relacionadas aos aspectos organizacionais incluem o engajamento da administração da instituição (itens 1, 3, 4, 7, 11 e 24), recursos organizacionais para segurança (itens $2,16,30,39$ ) e ênfase geral na segurança do paciente (itens 12 e 23$)^{(10)}$.

Os aspectos relativos à unidade de trabalho englobam apoio dos gerentes da unidade (itens 25,29 e 34), normas de segurança na unidade (itens 5, 8, 18, 32 e 40), reconhecimento da unidade e apoio à dedicação em relação à segurança (itens 10, 21, 22 e 31), aprendizado coletivo (itens 26, 35, 38 e 41), segurança psicológica (itens 27, 33 e 37) e responsividade aos problemas (itens 28 e 36$)^{(10)}$.

A categoria interpessoal envolve as dimensões medo do constrangimento (itens 6, 9, 43 e 44) e medo de culpabilização e punição (itens 15, 17 e 42). Outro aspecto importante para o clima de segurança foi considerado, mas 
não classificado nas categorias anteriores, que consiste na dimensão prestação de cuidado seguro, avaliado pelos itens 14 e $19^{(10)}$.

A escala de resposta às afirmativas é do tipo Likert com cinco pontos, cujas opções são: discordo totalmente (1), discordo (2), não concordo nem discordo (3), concordo (4) e concordo totalmente (5). O profissional pode ainda considerar a opção "não se aplica" (6) $)^{(9)}$. Nove itens $(6,9,14,15$, $17,34,42,43$ e 44) possuem conotação negativa e por isso devem ser invertidos para análise.

O escore é obtido de forma que se dê destaque às respostas que se opõem à segurança (1) discordo totalmente e (2) discordo, chamadas de respostas problemáticas. O cálculo pode ser feito para os itens, dimensões e para o clima de segurança geral. Primeiro, obtém-se o cálculo da porcentagem média das respostas problemáticas para cada item entre todos os respondentes. Em seguida, calcula-se a média de todas as médias dos itens em uma dimensão e a média de todos os itens no instrumento. Uma média baixa de porcentagem de respostas problemáticas indica melhor percepção do clima de segurança na instituição.

\section{Tradução e adaptação cultural}

Após o consentimento formal da autora do instrumento, professora e pesquisadora Dra. Sara Singer, seguiram-se os procedimentos metodológicos de tradução e adaptação do PSCHO recomendados pela literatura, com a finalidade de manter a mesma equivalência entre a versão original e a adaptada ${ }^{(13)}$.

Inicialmente, o instrumento foi traduzido por dois tradutores independentes, com fluência na língua inglesa, cuja língua materna era o português do Brasil. Um dos tradutores foi informado sobre os conceitos e objetivos do instrumento e o outro não teve acesso a essas informações.

As duas versões ( $\mathrm{T} 1$ e T2) foram analisadas por um terceiro tradutor independente, o que permitiu a comparação e o consenso de discrepâncias, obtendo-se uma versão síntese (T12). A T12 foi submetida à tradução de volta para o inglês (retrotradução), por dois tradutores com fluência em português, cuja língua materna era o inglês, produzindo duas versões (RT1 e RT2). Esses profissionais eram tradutores independentes, não fizeram parte da primeira fase da tradução e não foram informados sobre os conceitos mensurados pelo PSCHO.

Após a obtenção das retrotraduções, foi organizado um comitê de juízes composto por sete profissionais: uma médica docente, com experiência em cuidados intensivos e vivência no país de origem do instrumento; uma enfermeira, doutoranda e com experiência assistencial; uma enfermeira, doutoranda e docente na área de administração, com formação em Letras; uma gerente de enfermagem, com experiência na área de qualidade e segurança do paciente; duas docentes em enfermagem, pesquisadoras na área de segurança do paciente e uma docente de enfermagem, com experiência na tradução e adaptação de instrumentos.

Com o objetivo de analisar a validade de conteúdo e desenvolver a versão pré-final do PSCHO, todas as versões do instrumento ( $V O, T 1, T 2, T 12, R T 1$ e RT2) foram disponibilizadas aos juízes para uma avaliação quantitativa e qualitativa. Cada juiz recebeu uma carta-convite e instruções específicas, nas quais se solicitou a avaliação das equivalências semântica, idiomática, cultural e conceitual de cada item do instrumento.

A avaliação da equivalência semântica refere-se ao significado das palavras, em que se observa se palavras traduzidas têm o mesmo significado empregado na versão original ${ }^{(13-14)}$. A idiomática avalia se o item traduzido preserva o sentido da expressão na versão original ${ }^{(13)}$. A equivalência cultural refere-se à adequação de situações ou atividades abordadas que correspondem às situações vivenciadas no contexto cultural do Brasil, enquanto a conceitual diz respeito à análise de palavras com diferentes conceitos entre as culturas ${ }^{(13-14)}$.

A avaliação quantitativa do instrumento ocorreu de maneira individual, em que os membros do comitê atribuíram uma pontuação para a equivalência de cada item

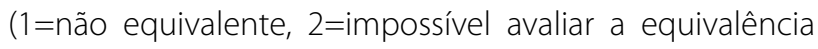
sem que o item seja revisto, 3=equivalente, mas necessi-

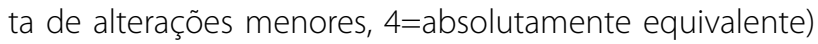
para o cálculo do Índice de Validade de Conteúdo (IVC). Este cálculo é feito levando-se em conta o número de juízes que pontuaram 3 ou 4, dividido pelo número total de juízes. Consideraram-se os itens com IVC menor que 0,80 elegíveis para revisão(15). A avaliação qualitativa realizou-se por meio de uma reunião com a maioria dos membros do comitê, na qual foram feitas sugestões sobre alguns aspectos do instrumento.

Com as adequações discutidas nesse encontro, obteve-se a versão pré-final do PSCHO, que foi submetida ao pré-teste em maio de 2017 com 40 sujeitos, entre eles enfermeiros, técnicos de enfermagem, fisioterapeutas e médicos. O critério para esta seleção partiu do princípio de que estes profissionais fazem parte da maioria dos que estão inseridos tanto na assistência direta ao paciente quanto em posições gerenciais de uma instituição hospitalar.

Os sujeitos receberam a versão pré-final do PSCHO e foram solicitados a avaliar o instrumento quanto à facilidade de compreensão dos itens e instruções, facilidade 
para assinalar as questões e o tempo dispendido para seu preenchimento.

O estudo foi aprovado pelo Comitê de Ética em Pesquisa da Unicamp (CAAE: 54036116.0.0000.5404) e todos os sujeitos assinaram o Termo de Consentimento Livre e Esclarecido.

\section{RESULTADOS}

O processo de tradução do instrumento $\mathrm{PSCHO}$ foi adequado e possibilitou a elaboração da síntese das versões T1 e T2, que posteriormente foi submetida à retrotradução. Na etapa de síntese (T12), a autora do instrumento foi consultada para esclarecer o significado de alguns termos, que apresentavam equivalência semântica diferente, mas que poderiam ser sinônimos na língua original (disciplined, punished e corrected). Após esclarecimentos, para os termos "disciplined" e "punished" considerou-se a tradução "punido" e, para "corrected", "corrigido". Em seguida, as versões $V O, T 1, T 2, T 12$, RT1 e RT2 foram submetidas à avaliação pelo comitê de juízes.

$\mathrm{Na}$ avaliação quantitativa, a maioria dos itens obteve uma concordância acima de $80 \%$ entre os juízes, o que demonstrou equivalência semântica, idiomática, cultural e conceitual dentre as versões do instrumento, conforme apresentado na Tabela 1.

Tabela 1 - Índice de validade de conteúdo dos itens da versão brasileira do Patient Safety Climate in Healthcare Organizations. Campinas, SP, 2017

\section{Itens}

IVC médio

Instruções, seção I, seção II, definição, opções, itens 1, 2, 3, 4, 5, 6, 7, 8, 10, 11, 12, 13, 14, 15, 19, $20,22,24,25,27,32,33,35,37,38,39,40,41,44,45,49,50$ e 51.

Título, itens 9, 21, 23, 34, 43 e 46

Itens 29, 30 e 42

Item 17

Itens 28, 31 e 48

Item 18

Item 36

Itens 16 e 26

Item 47

Fonte: Dados da pesquisa, 2017

Durante a reunião com os membros do comitê de juízes, momento em que foi possível realizar a avaliação qualitativa dos itens do instrumento, houve sugestões de alterações dos itens para adequar às equivalências semântica, idiomática, cultural e conceitual.

Entre os itens que obtiveram taxa de concordância menor que 80\%, o item 47 "Meu cargo é" apresentou discordância quanto à equivalência cultural, semântica e conceitual e, por se tratar de uma afirmativa que aborda categorias profissionais, sofreu modificações para adequar à realidade brasileira. No item 16 "Eu tenho tempo suficiente para realizar procedimentos com o paciente de forma segura" os juízes apontaram menor taxa de concordância quanto à equivalência semântica e cultural, tendo como sugestão a substituição da palavra "procedimentos" para "cuidado".

Para o item 26 "Os erros ocorridos em minha unidade têm levado a mudanças positivas no setor" apontou-se necessidade de subtração do termo "ocorri- dos em minha unidade" para melhor equivalência semântica e idiomática, uma vez que a versão original não especificava o local de ocorrência dos erros, mas sim se eles encorajavam mudanças na unidade. Dessa forma, as modificações sugeridas pelos juízes foram acatadas e o item assumiu a versão "Os erros têm levado a mudanças positivas em minha unidade/setor".

Além dos itens com taxa de concordância abaixo de $80 \%$, outras afirmativas foram modificadas. Os itens 46 e 48 sofreram alterações para adequação da equivalência cultural, pois abordam cargos e setores da unidade hospitalar, respectivamente, que devem ser adequados à realidade cultural do país.

Entre os termos padronizados, "administração/direção" foi utilizado sempre que a afirmativa se referisse ao gerenciamento da instituição (itens 3, 4, 7 e 11), enquanto que "gerência/coordenação" foi inserido nos itens relacionados à unidade, (itens 25, 28, 29, 34 e 37). A palavra "facility" apresentou divergência nos itens 23 e 39, assu- 
mindo a tradução "unidade". Após consenso, assumiu-se o significado de "instituição" para este termo, pois está presente em itens que representam dimensões de aspecto organizacional e não da unidade.

Além dessas afirmativas, o tempo verbal foi modificado no item 9 "Se eu cometer um erro que tenha consequências significativas e ninguém perceber, eu não irei revelar a ninguém sobre o ocorrido", assumindo o tempo presente no trecho "eu não revelo a ninguém sobre o ocorrido", e no item 30 "Eu recebi treinamento ade- quado para resolver problemas relacionados à segurança do paciente", cuja alteração ocorreu no trecho "Eu tenho recebido".

Com relação à substituição de palavras, no item 17 "Profissionais da área clínica que cometem erros graves, geralmente são punidos" o termo "área clínica" foi substituído por "assistência". No item 21 "Eu sou recompensado(a) pela rápida intervenção ao identificar um erro grave" a expressão "rápida intervenção" foi alterada para "agir rapidamente".

\begin{tabular}{|c|c|c|c|}
\hline Itens & Versão original & Síntese & Versão Pré-teste \\
\hline 18 & $\begin{array}{l}\text { In my unit, there is significant } \\
\text { peer pressure to discourage } \\
\text { unsafe patient care. }\end{array}$ & $\begin{array}{l}\text { Há uma pressão significativa dos colegas na } \\
\text { minha unidade para desencorajar práticas } \\
\text { que não sejam seguras ao paciente. }\end{array}$ & $\begin{array}{l}\text { Na minha unidade, há uma } \\
\text { pressão significativa dos colegas } \\
\text { para desencorajar práticas } \\
\text { inseguras ao paciente. }\end{array}$ \\
\hline 28 & $\begin{array}{l}\text { Bringing patient safety } \\
\text { problems to management's } \\
\text { attention usually results } \\
\text { in the problem being } \\
\text { addressed. }\end{array}$ & $\begin{array}{l}\text { Alertar o gestor sobre problemas com a } \\
\text { segurança do paciente geralmente leva à } \\
\text { resolução do problema. }\end{array}$ & $\begin{array}{l}\text { Alertar a gerência/coordenação da } \\
\text { minha unidade sobre problemas } \\
\text { com a segurança do paciente } \\
\text { geralmente leva à discussão } \\
\text { sobre o problema. }\end{array}$ \\
\hline 31 & $\begin{array}{l}\text { My performance is } \\
\text { evaluated against defined } \\
\text { safety standards. }\end{array}$ & $\begin{array}{l}\text { Meu desempenho é avaliado de acordo } \\
\text { com os padrões estabelecidos para a } \\
\text { segurança do paciente. }\end{array}$ & $\begin{array}{l}\text { Meu desempenho é avaliado } \\
\text { de acordo com padrões de } \\
\text { segurança definidos. }\end{array}$ \\
\hline 36 & $\begin{array}{l}\text { When I take time to } \\
\text { communicate about patient } \\
\text { safety problems there is } \\
\text { appropriate follow up. }\end{array}$ & $\begin{array}{l}\text { Quando dedico algum tempo para notificar } \\
\text { os problemas relacionados à segurança } \\
\text { do paciente, há um acompanhamento } \\
\text { apropriado aos pacientes. }\end{array}$ & $\begin{array}{l}\text { Quando dedico algum tempo para } \\
\text { notificar os problemas relacionados } \\
\text { à segurança do paciente, há um } \\
\text { seguimento apropriado. }\end{array}$ \\
\hline 42 & $\begin{array}{l}\text { I will be blamed if I make } \\
\text { an error. }\end{array}$ & $\begin{array}{l}\text { Serei responsabilizado se eu cometer um } \\
\text { erro. }\end{array}$ & $\begin{array}{l}\text { Serei considerado culpado se eu } \\
\text { cometer um erro. }\end{array}$ \\
\hline
\end{tabular}

Quadro 1 - Descrição dos itens alterados após avaliação do comitê de juízes Fonte: Dados da pesquisa, 2017.

Os demais itens que obtiveram equivalência menor que um e que sofreram modificações substanciais (em negrito) estão descritas no Quadro 1.

Após as modificações sugeridas pelos membros do comitê de juízes, seguiu-se a etapa do pré-teste, realizado em um hospital universitário com 40 profissionais, entre eles enfermeiros (12,5\%), técnicos de enfermagem (45\%), fisioterapeutas (2,5\%), médicos residentes (17,5\%), médicos docentes e assistentes (22,5\%), cujas áreas de trabalho eram: ambulatório, unidade de emergência referenciada (UER), unidade de terapia intensiva (UTI), centro de material esterilizado (CME), centro-cirúrgico (CC), enfermaria/ unidade de internação e de outras áreas. A maioria dos profissionais tinha entre 31 e 50 anos (72,5\%), era do sexo feminino (62,5\%), com mais de cinco anos de trabalho na instituição (70\%).
O tempo médio de preenchimento do PSCHO foi de 15,3 minutos (DP \pm 6 ), sendo o mínimo de sete e o máximo de 34 minutos. Embora 67,5\% dos sujeitos tenham avaliado como de fácil preenchimento, alguns sugeriram alterar a formatação da coluna para assinalá-las, com o intuito de destacar as opções existentes e tornar o preenchimento mais rápido. Quanto às instruções e às questões do $\mathrm{PSCHO}$, 85\% assinalaram ser de fácil compreensão.

O item 40 "Violações deliberadas dos procedimentos operacionais padrões são raras na minha unidade" apresentou concordância de 100\%, porém foi considerado por alguns sujeitos sem equivalência cultural pelo uso do termo "deliberadas". Para alterar este item, optou-se por consultar novamente os juízes e obteve-se o retorno da maioria, que concordou em subtrair o termo. Para o item 47, que trata das profissões, sugeriu-se alterar o 
enunciado "Meu cargo é" para "Minha função é", uma vez que a questão 46 já trata sobre o cargo ou posição ocupada pelo profissional na instituição.

Com as alterações feitas após o pré-teste, obteve-se a versão traduzida e adaptada do PSCHO para a cultura brasileira (Apêndice).

\section{口DISCUSSÃO}

O processo de tradução, síntese e retrotradução do instrumento PSCHO para a cultura brasileira efetivou-se de forma sistemática, de acordo com a literatura ${ }^{(13)}$. Apesar de ser um processo já bem estabelecido, cada etapa possui características específicas, que devem ser detalhadas pelo pesquisador. A tradução do instrumento é o primeiro passo para um trabalho bem executado, por isso é relevante selecionar criteriosamente os profissionais que farão parte dessa fase ${ }^{(16)}$.

A síntese das traduções exige um profissional especialista em linguística, para garantir que a equivalência semântica dos conceitos do instrumento, tanto na língua de origem, quanto na de destino, seja respeitada. Dois aspectos devem ser considerados nesta fase, o significado referencial (denotativo) e o geral (conotativo). A correspondência literal entre uma palavra na sua língua original e a sua respectiva tradução confere significado denotativo, porém sob o ponto de vista conotativo, uma palavra pode ter um impacto diferente no contexto cultural da população alvo(16).

Essa reflexão foi necessária no presente estudo, uma vez que na cultura de segurança do paciente a "punição" do profissional é compreendida como injusta e prejudicial, pois não analisa os fatores organizacionais para a ocorrência do erro(17). O uso deste termo indiscriminadamente poderia inverter o significado do item do instrumento. Dessa forma, o termo "corrected" não assumiu o significado punitivo, mas sim de redirecionamento da ação, alinhando à cultura de aprendizagem ${ }^{(18)}$.

A etapa de avaliação pelo comitê de juízes mostrou-se essencial para a adaptação e validade de conteúdo, pois considerou a experiência e vivência de cada especialista, o que colabora para a uniformização de termos, tornando os itens mais claros e de fácil compreensão na realidade brasileira ${ }^{(14,19)}$.

$\mathrm{Na}$ avaliação das equivalências do PSCHO pelo comitê de juízes foi possível observar maior divergência com relação à equivalência semântica. Assim como no estudo de adaptação transcultural do instrumento HSOPSC(20), algumas palavras foram modificadas e alguns termos padronizados com o intuito de facilitar a compreensão dos itens pelos sujeitos e mantê-los com o significado original.

A análise das equivalências mostrou que as etapas de adaptação cultural foram adequadas. Apesar de não ser consenso o número e a qualificação dos juízes ${ }^{(13,14)}$, considera-se crucial que estes profissionais sejam capazes de avaliar a qualidade das traduções, tomar decisões críticas, chegar a um consenso na existência de discrepâncias e consolidar todas as versões do instrumento(14). A presença de um profissional com experiência assistencial no país de origem do instrumento facilitou o consenso quando se discutiu a equivalência cultural de diversos itens, principalmente entre as opções de profissões e setores ("Minha função é" e "Área de trabalho", respectivamente) do PSCHO.

Embora o instrumento seja construído para aplicação junto aos diversos profissionais do cenário hospitalar, durante a seleção da amostra para o pré-teste optou-se por convidar sujeitos que representassem a população alvo do estudo, composta pela equipe de enfermagem, fisioterapeutas e médicos. Cada um deles contribuiu de forma qualitativa para melhor compreensão do instrumento e, de uma maneira geral, os sujeitos avaliaram de forma positiva as opções de resposta, compreensão dos itens e instruções da versão brasileira do PSCHO. Dos 51 itens, apenas o item 40 foi descrito como de difícil compreensão, indicando um adequado processo de adaptação cultural.

O tempo médio de preenchimento de 15 minutos pode ter sido influenciado pelo layout das opções de resposta da escala. Uma parcela dos sujeitos sugeriu alterações neste aspecto, com o intuito de facilitar a leitura de cada item e a localização da opção de resposta, o que foi acatado para facilitar o preenchimento do instrumento. Outra alteração sugerida e aprovada foi a substituição do enunciado do item "Meu cargo é" por "Minha função é", uma vez que cargo é uma posição, e não a profissão de alguém.

\section{— CONCLUSÃO}

O processo de tradução e adaptação do PSCHO para a cultura brasileira seguiu as etapas recomendadas internacionalmente e apresentou resultados satisfatórios com relação a sua compreensão pelos sujeitos. Como limitação, houve dificuldade de comparação dos dados com outras pesquisas, uma vez que o instrumento encontra-se traduzido apenas na China.

A versão do $\mathrm{PSCHO}$ obtida neste estudo corresponde à primeira etapa do processo de disponibilização de um instrumento numa cultura diferente da original em que foi desenvolvido. Estudos futuros devem ser elaborados para validação do questionário para fins de pesquisa. No ambiente da prática profissional, o uso do PSCHO poderá contribuir para a compreensão do clima de segurança do paciente e, assim, auxiliará na identificação das vulnerabilidades das instituições. Portanto, espera-se que esta seja uma ferramenta útil 
para os gestores direcionarem mudanças que efetivamente reduzam a um mínimo aceitável os riscos da assistência.

\section{口EFERÊNCIAS}

1. Makary MA, Daniel M. Medical error-the third leading cause of death in the US. BMJ. 2016;353:i2139. doi: https://doi.org/10.1136/bmj.i2139.

2. Mitchell I, Schuster A, Smith K, Pronovost P, Wu A. Patient safety incident reporting: a qualitative study of thoughts and perceptions of experts 15 years after 'To Err is Human'. BMJ Qual Saf. 2016;25(2):92-9. doi: https://doi.org/10.1136/ bmjqs-2015-004405.

3. Singer SJ, Vogus TJ. Reducing hospital errors: interventions that build safety culture. Annu. Rev. Public Health. 2013;34:373-96. doi: https://doi.org/10.1146/ annure v-publhealth-031912-114439.

4. Schwatka NV, Hecker S, Goldenhar LM. Defining and measuring safety climate: a review of the construction industry literature. Ann Occup Hyg. 2016;60(5):53750. doi: https://doi.org/10.1093/annhyg/mew020.

5. Braithwaite J, Herkes J, Ludlow K, Testa L, Lamprell G. Association between organisational and workplace cultures, and patient outcomes: systematic review. BMJ Open. 2017;7(11):e017708. doi: https://doi.org/10.1136/bmjopen-2017-017708.

6. Fan CJ, Pawlik TM, Daniels T, Vernon N, Banks K, Westby P, et al. Association of safety culture with surgical site infection outcomes. J Am Coll Surg. 2016;222(2):122-8. doi: https://doi.org/10.1016/j.jamcollsurg.2015.11.008.

7. Reis CT, Laguardia J, Vasconcelos AGG, Martins M. Reliability and validity of the Brazilian version of the Hospital Survey on Patient Safety Culture (HSOPSC): a pilot study. Cad Saúde Pública. 2016;32(11):e00115614. doi: https://doi. org/10.1590/0102-311x00115614.

8. Carvalho REFL, Cassiani SHB. Cross-cultural adaptation of the Safety Attitudes Questionnaire - Short Form 2006 for Brazil. Rev Lat-Am Enfermagem. 2012;20(3):8 telas. doi: https://doi.org/10.1590/S010411692012000300020.

9. Singer S, Meterko M, Baker L, Gaba D, Falwell A, Rosen A. Workforce perceptions of hospital safety culture: development and validation of the patient safety climate in healthcare organizations survey. Health Serv Res. 2007;42(5):19992021. doi: https://doi.org/10.1111/j.1475-6773.2007.00706.x
10. Benzer JK, Meterko M, Singer SJ. The patient safety climate in healthcare organizations (PSCHO) survey: short-form development. J Eval Clin Pract. 2017;23(4):853-9. doi: https://doi.org/10.1111/jep.12731.

11. Cochrane BS, Hagins M Jr, Picciano G, King JA, Marshall DA, Nelson B, et al. High reliability in healthcare: creating the culture and mindset for $\mathrm{pa}-$ tient safety. Healthc Manage Forum. 2017;30(2): 61-8. doi: https://doi. org/10.1177/0840470416689314.

12. Cunha MRPD. Adaptação cultural e avaliação das propriedades de medida do instrumento Patient Safety Climate in Healthcare Organizations [tese]. Campinas (SP): Universidade Estadual de Campinas; 2017.

13. Beaton D, Bombardier C, Guillemin F, Ferraz MB. Recommendations for the crosscultural adaptation of the DASH QuickDASH outcome measures. Toronto: Institute for Work \& Health; 2007 [cited 2017 Jun 20]. Available from: http://www.dash. iwh.on.ca/sites/dash/files/downloads/cross_cultural_adaptation_2007.pdf

14. Epstein J, Santo RM, Guillemin F. A review of guidelines for cross-cultural adaptation of questionnaires could not bring out a consensus. J Clin Epidemiol. 2015;68(4):435-41. doi: https://doi.org/10.1016/j.jclinepi.2014.11.021.

15. Alexandre NMC, Coluci MZO. [Content validity in the development and adaptation processes of measurement instruments]. Ciênc Saúde Coletiva. 2011;16(7):30618. Portuguese. doi: https://doi.org/10.1590/S1413-81232011000800006.

16. Reichenheim ME, Moraes CL. Operationalizing the cross-cultural adaptation of epidemological measurement instruments. Rev Saúde Pública. 2007;41(4). doi: https://doi.org/10.1590/S0034-89102006005000035.

17. Urbanetto JS, Gerhardt LM. Patient safety in the healthcare education research triad [Editorial]. Rev Gaúcha Enferm. 2013;34(3):12-3. doi: https://doi. org/10.1590/S1983-14472013000300001.

18. Singer SJ, Benzer JK, Hamdan SU. Improving health care quality and safety: the role of collective learning. J Healthc Leadersh. 2015;7:91-107. doi: https://doi. org/10.2147/JHL.S70115.

19. Dalla Nora CR, Zoboli E, Vieira MM. Validation by experts: importance in translation and adaptation of instruments. Rev Gaúcha Enferm. 2017;38(3):e64851. doi: https://doi.org/10.1590/1983-1447.2017.03.64851.

20. Reis (T, Laguardia J, Martins M. Adaptação transcultural da versão brasileira do Hospital Survey on Patient Safety Culture: etapa inicial. Cad Saúde Pública. 2012;28(11):2199-2210. doi: https://doi.org/10.1590/S0102$311 \times 2012001100019$.
Autor Correspondente:

Edinêis de Brito Guirardello E-mail: guirar@unicamp.br
Recebido: 06.02.2018

Aprovado: 02.07.2018 


\title{
APÊNDICE
}

\section{CLIMA DE SEGURANÇA DO PACIENTE EM INSTITUIÇÕES DE SAÚDE}

\author{
PARTE I \\ Este conjunto de afirmativas corresponde às suas experiências relacionadas à segurança do paciente na sua unidade de trabalho e na sua \\ instituição atualmente, salvo as observações em contrário. \\ Algumas afirmativas referem-se à "minha unidade". Médicos e outros prestadores de cuidados que não estão alocados em uma única unidade \\ devem respondê-las com base nas experiências adquiridas por meio de seus serviços prestados, tais como assistência clínica ou cirúrgica. Todos \\ os outros profissionais devem responder essas afirmativas segundo suas experiências na unidade de trabalho em que passam a maior parte do \\ tempo, como em uma Unidade de Terapia Intensiva, Centro Cirúrgico ou Ambulatório.
}

DEFINIÇÃO: Segurança do Paciente - Ações para evitar, prevenir ou corrigir eventos adversos que podem ser decorrentes do processo de prestação de cuidados de saúde.

INSTRUÇÕES: Para as afirmações a seguir, por favor, assinale se você "discorda totalmente", "discorda", "não concorda nem discorda", "concorda" ou "concorda totalmente". Caso você queira alterar alguma resposta, preencha o quadrado que corresponde à sua nova opção e faça um círculo em volta dele.

PREENCHIMENTO CORRETO $\square \square \square \times \square \square$

1. Existe um bom fluxo de comunicação entre os níveis hierárquicos, ascendente e descendente, sobre os assuntos relacionados à segurança do paciente.

2. Tenho à minha disposição recursos adequados (pessoal, orçamento e equipamento) para prestar cuidado seguro ao paciente.

3. A administração/direção da instituição apoia um clima que promove a segurança do paciente.

4. A administração/direção da instituição tem uma visão clara dos riscos associados ao cuidado do paciente.

5. Minha unidade empenha-se em identificar e avaliar os riscos para garantir a segurança do paciente.

6. Pedir ajuda é um sinal de incompetência.

7. A administração/direção da instituição tem conhecimento dos tipos de erros que realmente ocorrem nesta instituição.

8. Minha unidade faz um bom trabalho de gerenciamento de riscos para garantir a segurança do paciente.

9. Se eu cometer um erro que tenha consequências significativas e ninguém perceber, eu não revelo a ninguém sobre o ocorrido.

10. Minha unidade reconhece as conquistas individuais relacionadas à segurança por meio de recompensas e incentivos.

11. A administração/direção da instituição considera a segurança do paciente quando são discutidas mudanças nos processos internos.

12. Comparada com outras instituições na área, esta instituição preocupa-se mais com a qualidade do serviço que presta ao paciente.

13. Eu tenho aprendido como melhorar meu próprio trabalho ao tomar conhecimento dos erros cometidos pelos meus colegas.

14. No último ano, eu presenciei um colega de trabalho fazer algo que me pareceu ser inseguro para o paciente.

15. Se as pessoas descobrirem que eu cometi um erro, eu serei punido.

16. Eu tenho tempo suficiente para realizar os cuidados ao paciente de forma segura.

17. Profissionais da assistência que cometem erros graves, geralmente são punidos.

18. Na minha unidade, há uma pressão significativa dos colegas para desencorajar práticas inseguras ao paciente.

19. Eu nunca presenciei um colega de trabalho fazer algo que me parecesse inseguro para o cuidado do paciente.

20. No último ano, eu fiz algo que não foi seguro para o paciente.

21. Eu sou recompensado (a) por agir rapidamente ao identificar um erro grave.

22. Minha unidade oferece treinamento sobre o trabalho em equipe, a fim de melhorar o desempenho no cuidado $e$ segurança do paciente.

23. Em geral, o nível de segurança do paciente nesta instituição está melhorando.

24. As decisões relacionadas à segurança do paciente são tomadas pelas pessoas mais qualificadas, independentemente do cargo ou hierarquia.

25. A gerência/ coordenação da minha unidade me ajuda a superar problemas que dificultam a prestação de cuidado seguro ao paciente. 
INSTRUÇÕES: Para as afirmações a seguir, por favor, assinale se você "discorda totalmente", "discorda", "não concorda nem discorda", "concorda" ou "concorda totalmente". Caso você queira alterar alguma resposta, preencha o quadrado que corresponde à sua nova opção e faça um círculo em volta dele.

26. Os erros têm levado a mudanças positivas em minha unidade/ setor.

27. A equipe sente-se confortável para questionar as ações daqueles com maior autoridade quando a segurança do paciente está em risco.

28. Alertar a gerência/ coordenação da minha unidade sobre problemas com a segurança do paciente geralmente leva à discussão sobre o problema.

29. Na minha unidade, a gerência/ coordenação coloca a segurança em um nível mais elevado de importância do que o cumprimento do cronograma e da produtividade.

30. Eu tenho recebido treinamento adequado para abordar problemas relacionados à segurança do paciente.

31. Meu desempenho é avaliado de acordo com padrões de segurança definidos.

32. Na minha unidade, qualquer pessoa que intencionalmente viole os padrões ou regras de segurança é corrigida.

33. A equipe manifesta-se com liberdade caso observe algo que pode afetar negativamente o cuidado ao paciente.

34. Sempre que a pressão aumenta, a gerência/coordenação da minha unidade quer que trabalhemos mais rápido, mesmo que isso implique em abreviar tarefas, o que pode afetar a segurança do paciente de forma negativa.

35. Na minha unidade, identificamos e resolvemos problemas de segurança antes que o incidente de fato ocorra.

36. Quando dedico algum tempo para notificar os problemas relacionados à segurança do paciente, há um seguimento apropriado.

37. Eu me sinto confortável em informar minhas preocupações em relação à segurança, sem medo de ser punido pela gerência/coordenação da minha unidade.

38. Nosso processo de investigação de acidentes e incidentes é efetivo na identificação das causas-raízes.

39. Essa instituição dispõe de recursos suficientes para o acompanhamento dos problemas de segurança identificados.

40. Violações dos procedimentos operacionais padrões são raras na minha unidade.

41. Na minha unidade, os problemas e erros relacionados à segurança do paciente são comunicados às pessoas certas para que o problema seja corrigido.

42. Serei considerado culpado se eu cometer um erro.

43. As pessoas duvidarão das minhas habilidades se eu fizer perguntas.

44. Meus colegas de trabalho perderão o respeito por mim se eles souberem que cometi um erro.

45. Eu me sinto envergonhado quando cometo um erro na frente dos meus colegas de trabalho.

\section{PARTE II}

Por favor, complete as seguintes informações. Lembre-se de que suas respostas são anônimas.

46. Eu sou: $\square$ Administrador/ Diretor da instituição. $\square$ Gerente/ Coordenador da unidade. $\square$ Não ocupo nenhum dos cargos.

47. Minha função é:

$\square$ Médico

$\square$ Residente

$\square$ Enfermeiro

$\square$ Aux. Enfermagem

$\square$ Assistente Social

$\square$ Terapeuta Ocupacional

$\square$ Fonoaudiólogo

Farmacêutico

$\square$ Odontologista

Fisioterapeuta

$\square$ Psicólogo

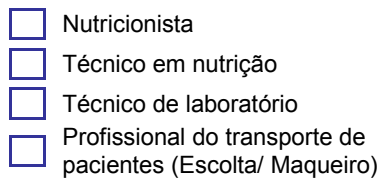

$\square$ Auxiliar de limpeza

$\square$ Técnico de radiologia

$\square$ Auxiliar administrativo

$\square$ Téc. Enfermagem

(Assinale apenas UMA

$\square$ Ambulatório

$\square$ Pronto-Socorro/ Unidade de Emergência

Referenciada

$\square$ Unidade de terapia intensiva

$\begin{array}{ll}\text { 49. Idade } & \square \quad 18-25 \text { anos } \\ \text { 50. Sexo } & \square \text { Feminino }\end{array}$

51. Há quanto tempo você trabalha nesta instituição?

\section{$\square$ Centro de Material \\ $\square$ Centro cirúrgico \\ $\square$ Laboratório}

$\square \quad 26-30$ anos Masculino

0 - 6 meses pacientes (Escolta/ Maqueiro) $\square \square \square \square \square$ 